\title{
Review
}

Ophthalmologica

\section{Retinal Folds following Retinal Detachment Surgery}

\author{
Heinrich Heimann $^{\text {a }}$ Silvia Bopp ${ }^{b}$ \\ ${ }^{a}$ Royal Liverpool University Hospital, Liverpool, UK; ${ }^{b}$ Augenklinik Universitätsallee, Bremen, Germany
}

\section{Key Words}

Retinal detachment $\cdot$ Retinal folds $\cdot$ Scleral buckling

surgery $\cdot$ Primary vitrectomy $\cdot$ Complications

\begin{abstract}
Purpose: To review the occurrence of macular retinal folds following retinal detachment surgery. Method: Review of the literature. Results: Macular folds have been reported in up to $3 \%$ of cases postoperatively. Risk factors are tamponading gas bubble, large exoplants, detachments with bullous configuration or running through the fovea, and incomplete drainage of subretinal fluid. Preventive measures include correct posturing and avoidance of modifiable risk factors. The natural history is variable. Complete resolution as well as persisting folds with permanent structural damage have been reported. Treatment indications and techniques remain controversial. Very few successful reports of surgical correction have been published to date. Conclusions: Macular folds are a serious but underreported complication of retinal detachment surgery that can be avoided in the majority of cases. Macular folds can be prevented by correct posture and avoiding large exoplants, large tamponade bubbles and incomplete drainage of subretinal fluid.
\end{abstract}

Copyright $\odot 2011$ S. Karger AG, Basel
(C) 2011 S. Karger AG, Basel

0030-3755/11/2265-0018\$38.00/0

Fax +41613061234

E-Mail karger@karger.ch

www.karger.com
Accessible online at:

www.karger.com/oph

\section{Introduction}

Retinal folds are a rare but potentially severe complication following retinal detachment surgery. They can not only occur after scleral buckling surgery (fig. 1) and primary vitrectomy (fig. 2), but rarely also after pneumatic retinopexy if an internal gas tamponade is employed. In this article, we review their pathophysiology, strategies to prevent their occurrence, and management options.

\section{Incidence}

Retinal folds at the posterior pole are probably an underreported complication. In contrast, retinal folds in the periphery can be seen more commonly (for example, radial folds on episcleral buckles) but are usually not considered to be of significant clinical impact as long as they are not associated with persistent or recurrent retinal detachments. To date, there are only few publications on the topic of macular retinal folds following rhegmatogenous retinal detachment (RRD) surgery. These are mainly individual case reports [1-6]. Only a small number of case series have been published [7-9]. One publication com- 
Fig. 1. Macular fold (long arrow) following scleral buckling surgery with gas tamponade using a large radial exoplant for a tractional horseshoe tear in the superior nasal quadrant. Because the optic nerve head serves as a fixation point, a double-arcuated configuration of the nasal (double arrow) and temporal part (long arrow) of the fold has occurred.

Fig. 2. Macular fold following primary vitrectomy. a Preoperative fundus drawing showing that the detachment extends to the macula. Visual acuity $=0.1$. b Postoperative optical coherence tomography that shows some hyperreflective material underneath the retinal fold. c Postoperative clinical picture of the macular fold that has lead to a distortion of the temporal vessel arcades. Visual acuity $=0.2$, associated with severe metamorphopsia and binocular confusion.
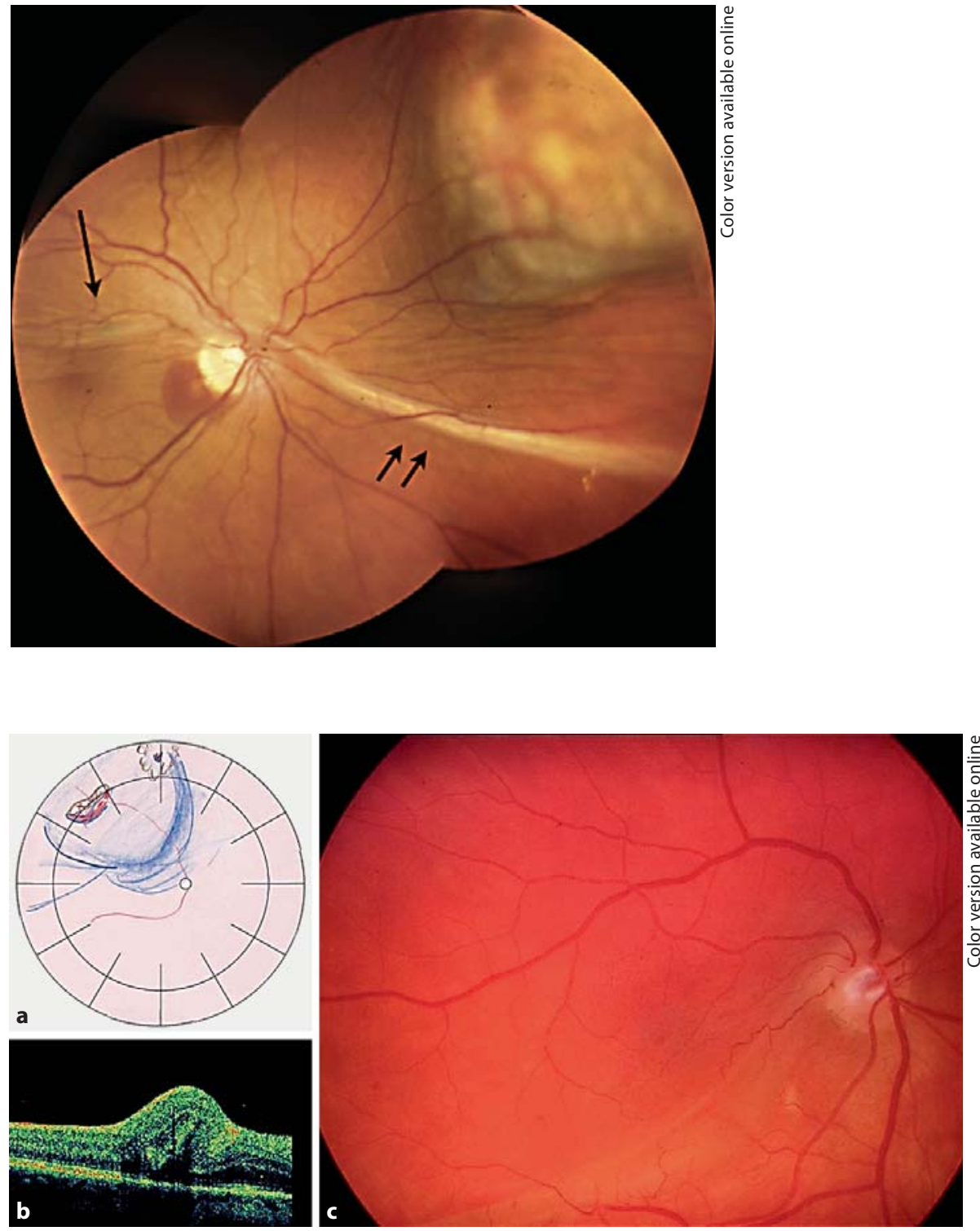

mented on the incidence in a consecutive series of 137 patients treated with scleral buckling surgery [10]. The authors found a relatively high number of macular folds in 4 of 136 cases (2.8\%).

The paucity of scientific reports, in particular in recent years, is in sharp contrast to our clinical experience. Almost every vitreoretinal surgeon can recall one or more cases of central retinal folds following RRD surgery and has his or her personal strategy towards their prevention or treatment [pers. commun., annual meeting of the BEAVRS 2010, Newport, UK]. With the increasing trend towards day-case surgery for retinal de- tachments, one could speculate that we may see a rise in occurrence of this complication in the near future as appropriate posture of the patients cannot always be sustained during the important hours immediately after the surgery [2].

\section{Pathophysiology}

In $\mathrm{RRD}$, the neurosensory retina is separated from the retinal pigment epithelium by an influx of aqueous humour and liquefied vitreous through a retinal break. The 
aims of the surgical treatment are to seal the retinal break and to remove the fluid from the subretinal space in order to restore the apposition of photoreceptors and retinal pigment epithelium. Ideally, the photoreceptors will reattach themselves in a position corresponding to the location before the detachment. However, autofluorescence studies suggest that a minor, clinically insignificant displacement of retinal tissue occurs in the majority of cases following successful RRD repair [11].

The retinal displacement is caused by the mobility and elasticity of the detached retina. An influx of subretinal fluid stretches the retina and can increase its surface. Retinal redundancy may be increased if scleral exoplants are used. If an intraocular tamponade is injected, the buoyancy of the intraocular gas bubble will force the detached retina back to the retinal pigment epithelium and choroid. This can result in creasing of retinal tissue at the edges of the tamponade bubble, which can routinely be seen immediately after retinal reattachment even if no tamponade is installed (for example, radial folds on circumferential retinal buckles following external drainage of subretinal fluid). The majority of these retinal folds will disappear within a relatively short period of time and the apposition of photoreceptors to retinal pigment epithelial cells is restored.

Central retinal folds are usually caused by medium- to large-sized intraocular gas bubbles that push residual subretinal fluid towards the posterior pole. As a result of incomplete internal or external drainage, a relatively small amount of subretinal fluid is trapped under the retina. Retinal tissue with remaining subretinal fluid is squeezed between the buoyant tamponade bubble and the attached retina. Once the retinal break is sealed by the internal tamponade, the subretinal fluid will be reabsorbed into the choroid. If this happens slowly or over a larger surface (e.g. when only a small tamponade bubble or a large amount of subretinal fluid are present), the retinal surface will decrease over time to match that of the underlying tissue. As a result, a smooth retinal reattachment will occur, and peripheral retinal folds may slowly disappear. However, retinal tissue may also be acutely 'pinched' between the buoyant tamponade bubble and adjacent retina (fig. 3,4$)$. The trapped subretinal fluid at the edge of the tamponade bubble acts like a hand in the glove that is then removed, leaving behind a retinal duplication with a base-to-base apposition of the photoreceptor layers. After a relatively short period of time, the folds may appear to be fixed and result in malfunction and injuries to the involved and neighbouring tissue. The ensuing structural and functional effects are:
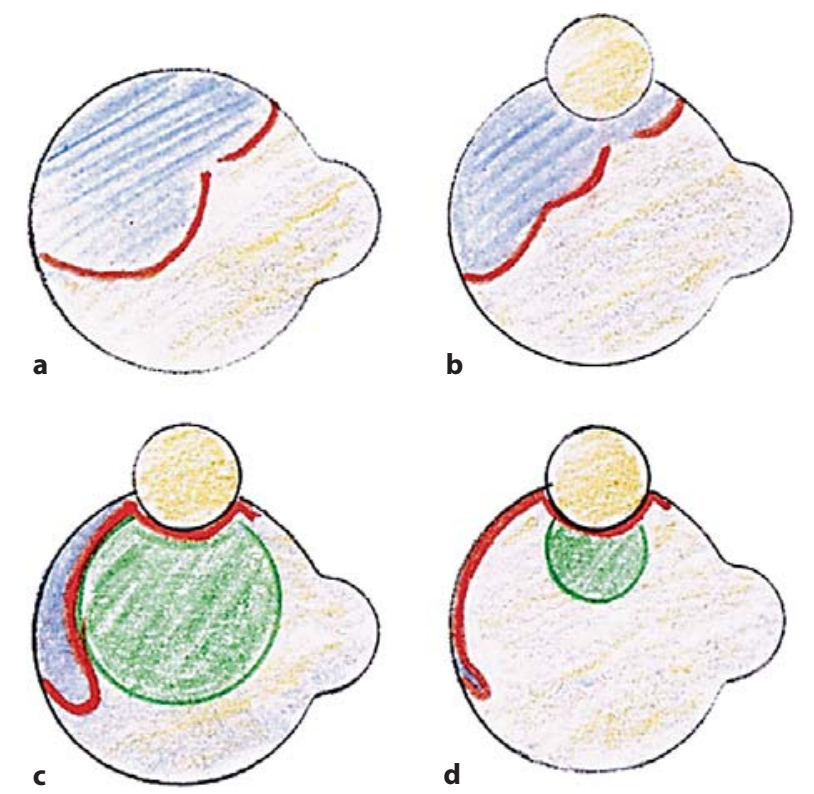

Fig. 3. Formation of a retinal fold following scleral buckling surgery. a Bullous detachment running through the macula. b The scleral buckle is placed in the area of the retinal break. Fluid is shifted towards the posterior pole. There is redundant retina due to the indenting effect of the buckle. $\mathbf{c}$ The intraocular gas bubble tamponades the break. It also pushes the detached retina against the retinal pigment epithelium and shifts fluid centrally. Subretinal fluid collects at the border of the gas bubble and preforms a retinal fold at the inferior edge of the bubble. $\mathbf{d}$ The subretinal fluid reabsorbs and leaves a retinal fold with a photoreceptor-tophotoreceptor apposition at the macula.

- loss of phototransduction in the photoreceptors that are separated from the retinal pigment epithelium;

- apoptosis and thinning of the photoreceptor layer within the fold [12];

- visual field defect caused by the redundant tissue that covers the neighbouring retina;

- metamorphopsia caused by the distortion of the fold and the adjacent retina.

Symptoms caused by the retinal folds depend on their location and extent as well as the distortion of the adjoining retinal tissue. When the macular area is involved, visual loss and metamorphopsia typically occur $[2,8]$. The fold can also cause a scotoma that may be perceived as a line across the central visual field $[1,3,6]$. 


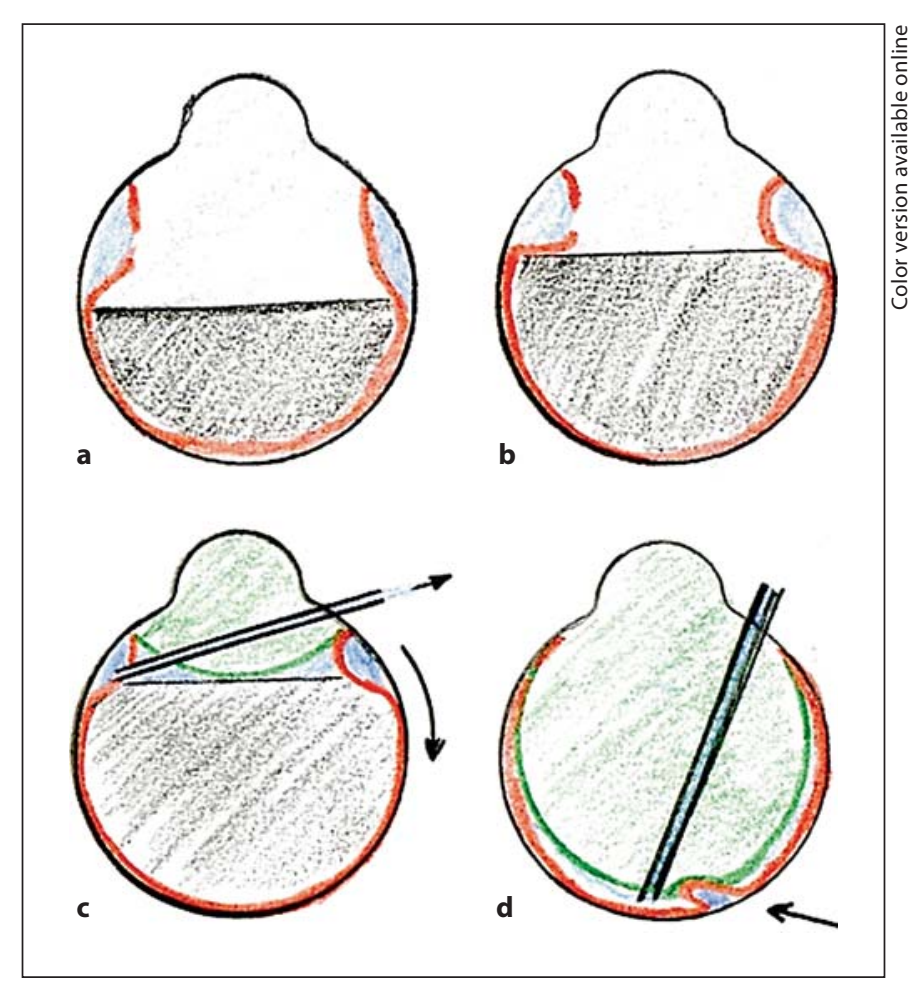

Fig. 4. Formation of a retinal fold following primary vitrectomy. a Heavy liquid reattaches the central retina and pushes subretinal fluid anteriorly. b Most of the subretinal fluid is drained internally through the break. However, some subretinal fluid, in particular in areas away from the break, is pushed into the subretinal space anterior to the break in a doughnut configuration. c Fluid air exchange. The air pushes the subretinal fluid down towards the break where it can be drained with a fluid needle. However, some fluid, in particular in the areas away from the retina, can bypass the internal drainage and will be shifted towards the posterior pole with increasing influx of air. $\mathbf{d}$ The wave of subretinal fluid has reached the posterior pole, where it is now trapped by the air bubble pressing against the retina. The result is a compression fold at the macula.

\section{Risk Factors}

Risk factors for the formation of retinal folds are:

- Use of an intraocular gas tamponade - this is one of the key elements in the formation of macular folds; they seem not to occur without an internal tamponade [9].

- Recent onset of retinal detachment [10] - subretinal fluid has a relatively low viscosity and cellularity, resulting in a quicker reabsorption of subretinal fluid. In addition, the retina is more elastic compared with a relatively stiffer retina in long-standing cases. If the subretinal fluid has a higher viscosity, which can often be seen in long-standing RRD, reabsorption of subretinal fluid may be delayed. This gives more time for the retina to reattach itself smoothly. Moreover, after a prolonged reabsorption of subretinal fluid, the edge of the tamponade bubble (where the fold forms) may already be further out in the periphery due to decreased size of the tamponade bubble.

- Large and circumferential buckles $[5,9,10]$ - the larger the buckle, the smaller the intraocular volume and the more redundant retina is there to form a fold. In most cases described after scleral buckling surgery, a circumferential exoplant either alone or in combination with a radial exoplant was used, whereas macular folds following a single small radial implant seem to be a rarity [9].

- Superior bullous detachment $[5,9,10]$ - the detached retina hangs like a saggy sail in the vitreous cavity. In the usual upright position, the gas bubble reattaches the superior part of the detachment and pushes the subretinal fluid inferiorly, where a compression fold may form at the inferior edge of the tamponade bubble.

- External drainage of subretinal fluid - in scleral buckling surgery, almost all cases of retinal folds occur after transscleral drainage of subretinal fluid $[9,10]$. This may not occur if a larger amount of subretinal fluid is left behind initially as this leaves more fluid volume in the preformed fold. A broader fold and distribution of the pressure from the tamponade bubble over a larger area of subretinal fluid will delay and spread the absorption of the subretinal fluid over a larger area.

- Incomplete internal drainage of subretinal fluid, in particular when performing primary vitrectomy [8] - drainage of larger amounts of subretinal fluid in combination with an internal tamponade may trap a thin layer of residual subretinal fluid in a fold at the inferior edge of the detachment.

- Retinal detachment running through the fovea $[8$, 9] - a predilection site of fold formation is along the edge of the primary detachment (fig. 2, 5). This line may act like a tether against which the subretinal fluid is pushed and where it gets caught to form the retinal fold [8]. However, folds can also appear in retinal areas that were initially not affected by the retinal detachment. It may also be that the optic disc forms a point of fixation and anchor for the macular fold (fig. 1) [12]. Without this, the retinal fold may be pushed to areas outside the fovea. 


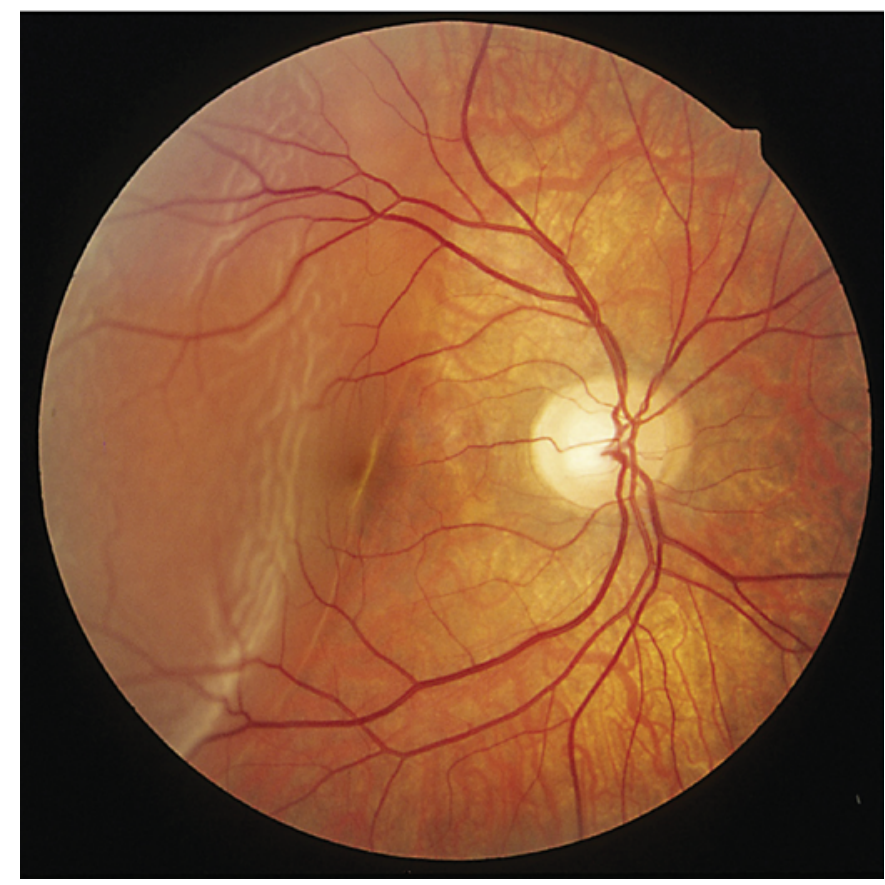

Fig. 5. Risk factor for developing a postoperative macular fold: retinal detachment running through the fovea.

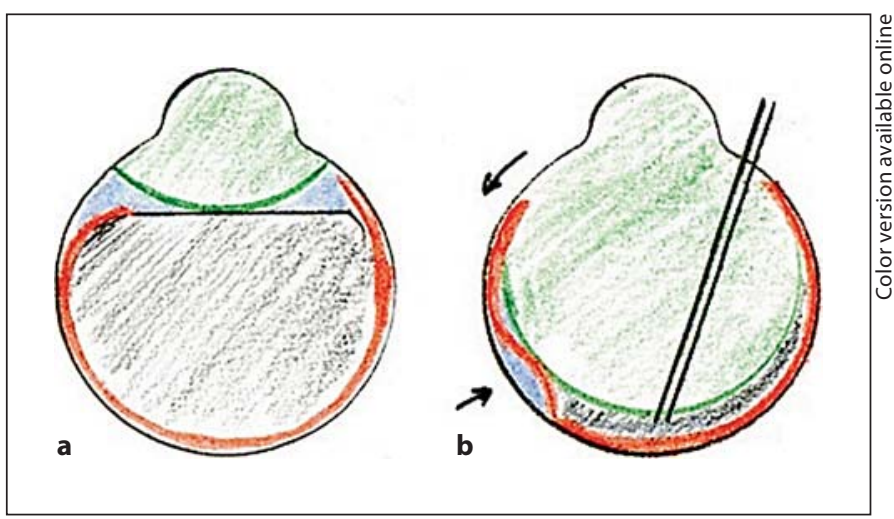

Fig. 6. Formation of a retinal fold following primary vitrectomy for a giant tear. a Slippage occurs if, at the end of the procedure during fluid air exchange, water is present in between the heavy liquid below and the air above. The retinal edge 'lies down' on the heavy liquid bubble. This opens the subretinal space and a wedge of fluid shifts under the retina. $\mathbf{b}$ The anterior edge of the tear is pushed back against the retinal pigment epithelium. The wave of subretinal fluid is pushed towards the posterior pole. Slippage with a macular fold at the posterior pole may occur.
- Slippage of the retina following retinal detachment with a giant retinal tear (fig. 6) [13].

A good example of how macular folds can be created is limited macular translocation for exudative age-related macular degeneration [12]. The combination of an induced recent-onset retinal detachment, retinal redundancy following scleral shortening, gas tamponade and a border of the retinal detachment running through the fovea resulted in the creation of retinal folds in all cases, and persistent macular folds as a serious complication in $4 \%$ in a series of 153 eyes reported on by Fuji et al. [14].

\section{Diagnosis and Additional Investigations}

Retinal folds are formed within the first hours following RRD surgery. With careful indirect ophthalmoscopy, they can be detected on the first postoperative day $[3,10]$. In a series of 32 patients reported on by Larrison et al. [8], the diagnosis was established between 3 days and 2.5 months postoperatively, with a median time to diagnosis of 10 days. One of the reasons for a delayed diagnosis is that indirect ophthalmoscopy is challenging with a gas tamponade in situ. In addition, visual symptoms are not evident due to the high refractive error and low visual acuity associated with an intraocular gas tamponade.

The diagnosis is usually established by indirect ophthalmoscopy. The differential diagnosis includes subretinal strands associated with proliferative vitreoretinopathy, a demarcation line, epiretinal membrane formation, choroidal folds and retinal incarceration [10]. Recent publications have illustrated optical coherence tomography findings of retinal folds (fig. 2) $[2,4,6]$. These include a discontinuity at the inner segment-outer segment junction and an indentation of the overlying retinal layers [4]. A thickening of the inner limiting membrane and corresponding vitreoretinal adhesions have been described by the same group. Optical coherence tomography may also be a useful tool to measure the dimensions of the fold, its relation to the fovea and possible changes over time [6]. With fundus autofluorescence, the retinal fold appears slightly hyperautofluorescent compared with the surrounding tissue [4].

\section{Prevention}

The major risk factors for developing macular folds are well defined. A significant number of these risk factors can be influenced by modifications of the surgical tech- 
nique. In cases with non-modifiable risk factors (retinal detachment across the macula, bullous detachment), the emphasis should be placed on optimisation of the surgical technique and the correct postoperative posture. With these measures, most retinal folds should be avoidable [5].

\section{Surgical Technique}

Scleral Buckling Surgery with Intravitreal Gas as an Adjunct

The three key factors that may promote the formation of macular folds following scleral buckling surgery are (1) the size of the injected tamponade bubble, (2) drainage of subretinal fluid and (3) the size of the exoplant (fig. 3). If possible, the injection of an intraocular tamponade bubble should be avoided. If deemed necessary (e.g. strong traction on the tear flap, fishmouth phenomenon), only a small bubble (around $1 \mathrm{ml}$ ) should be injected and strict positioning assured. In contrast to primary vitrectomy, only short-term tamponade of the break for a few hours is needed [9]. Large scleral buckles or a combination of multiple buckles should be avoided in patients at risk, as well as external drainage. This depends on the skill and training of the surgeon.

Overall, the problem of retinal folds following this type of surgery seems to have minimised over the past years. This is a result of the increasing popularity of primary vitrectomy in more challenging cases of retinal detachment. Advanced scleral buckling surgery with multiple buckles, injection of an intraocular tamponade and subretinal drainage has become a rarity.

\section{Primary Vitrectomy}

The key factors in preventing retinal folds following primary vitrectomy are (1) complete drainage of the subretinal fluid and (2) avoidance of submacular displacement of fluid during fluid air exchange. In the majority of cases, an internal drainage is performed through one of the preexisting retinal breaks. Situations that may promote incomplete drainage of subretinal fluid are small or undetected breaks, highly viscous subretinal fluid and an awkward position of the retinal break that may be difficult to reach with the fluid needle, in particular in phakic eyes. Sufficient drainage is easily performed through very anterior breaks (with the use of heavy liquids) or posterior breaks (with passive/active suction during fluid air exchange). However, the majority of breaks are somewhere in between at the equator. One problem can be that with the aid of heavy liquid, a significant amount of subretinal fluid may be anteriorly displaced into the periph- ery. Although most of it will be drained internally through the retinal break, residual subretinal fluid may be pushed into a doughnut shape under the anterior retina (fig. 4). The surgeon may not even be aware of this. During final fluid air exchange, this doughnut of subretinal fluid is then again pushed centrally. Once it has passed the most posterior break, it is trapped in the subretinal space and displaced under the central retina. This can cause a steamroller effect which will promote the formation of retinal folds and may convert a macula-on into a maculaoff retinal detachment.

To avoid these problems, the surgeon should aim at a complete drainage of the subretinal fluid. If the break is small and the subretinal fluid very viscous, the break can be slightly enlarged. If the break is difficult to reach or the subretinal fluid trapped in an area away from the break, a small anterior retinotomy close to the ora serrata can be placed. With the use of heavy liquid, this will greatly facilitate the drainage of the subretinal fluid. If subretinal fluid is trapped in a doughnut shape under the anterior retina, one initially fills the vitreous cavity with heavy liquid up to the posterior edge of the most central break. Then, a fluid air exchange is performed just in the area above the heavy liquid. The subretinal fluid is 'sandwiched' between the air above and the heavy liquid below. Increasing influx of air will push the anterior subretinal fluid down to the level of the heavy liquid, where it can be drained through the retinal break. Using a bimanual technique, indentation with a scleral depressor towards the break may assist in 'milking' the subretinal fluid internally through the break.

In cases with large breaks or retinotomies, slippage and displacement of subretinal fluid can occur during fluid air exchange. This can be avoided if all water is eliminated from the vitreous cavity by a complete heavy-liquid fill up to the level of the infusion line, as described by Wong et al. [13].

\section{Posture}

One of the key concepts to avoid retinal folds regardless of the surgical approach is the correct postoperative posture of patients at risk [5]. Although there is a consensus on how retinal folds are formed, there are diverging and sometimes conflicting ideas on the correct posture to counteract the formation of retinal folds:

- Prone position (face down) during the first postoperative hours $[8,15]$ - the buoyancy of the gas bubble in this posture should promote a smooth attachment of the posterior pole. At the same time, the subretinal fluid is displaced down into the periphery and will 
either be absorbed through the retinal pigment epithelium or drained internally into the vitreous cavity through the peripheral retinal break. After a few hours, the posture is changed according to the position of the break, for example sitting up for breaks in the superior periphery. If the position is changed slowly towards the position that puts the break in the least dependent position, this is also referred to as the 'steamroller technique'. One argument against the recommendation of postoperative positioning is that the steamroller effect may also trap subretinal fluid at the posterior pole. If the face-down position is taken immediately after surgery, the buoyant tamponade bubble may act like a balloon inside the eye that pushes and fixes the fold at the posterior pole against the underlying surface. Macular folds have been described following face-down positioning during the immediate postoperative period [2].

- Supine position (flat on the back) during the first hours - the idea behind this position is that the peripheral breaks are immediately covered by the tamponade. At the same time, there is no gas bubble pressing against the retina at the posterior pole as a layer of fluid should already be present (or may be left behind intentionally). Therefore, no fold should be 'pinched' into the retina in this area. The reabsorption of the subretinal fluid into the choroid takes place unhindered by a bubble pressing against the subretinal fluid, enabling a smooth reattachment of the retina. Potential folds are formed at the edge of the tamponade bubble outside the macula. As with the 'face-down' recommendation mentioned above, the position is changed after a few hours according to the location of the retinal break(s).

- Posture according to the location of the retinal break - this corresponds in part to the theory of prone positioning as described above. It follows the observation that in a significant number of cases with retinal folds, the patients were postured with the break in the most superior position postoperatively $[8,9]$. The underlying thought is that if the break is covered by the tamponade, the formation of retinal folds is encouraged when the gas bubble compresses all or almost all of the area of retinal detachment. In order to avoid this, the patient should not be positioned with the break in the least dependent location during the first hours after surgery (e.g. left cheek-to-pillow positioning should be avoided with a temporal break in the right eye).

The authors believe that following scleral buckling surgery with intravitreal gas injection, the steamroller technique carries the best chance to prevent macular folds. Following primary vitrectomy, careful observation of subretinal fluid shifting towards the posterior pole during endodrainage and final gas tamponade is mandatory. If a minor amount of fluid is observed sliding towards the macula, it is sufficient to keep the posterior pole covered by a small puddle of preretinal fluid. Postoperatively, the patient should then stay supine for about $2 \mathrm{~h}$ to allow the subretinal fluid to be reabsorbed. As retinal defects most likely are located peripherally, this position does not interfere with an appropriate tamponade of the causative hole(s). Thereafter, the patient can be advised to posture according to the location of the break(s). In cases in which a substantial 'wave' of subretinal fluid can be seen moving towards the posterior pole, the fluid air exchange should be stopped immediately. Repeated manoeuvres to drain the subretinal fluid as outlined above must then be performed.

\section{Management}

Once a retinal fold has formed, recommendations regarding its management vary significantly. This is because the natural course is variable and difficult to predict for the individual case. On the one hand, a relatively large number of patients with spontaneous improvement have been illustrated in the literature, and one may be inclined to watch and wait first. On the other hand, permanent damage may occur very early after fold formation, and techniques to deal with such a complication are available due to improvements in instrumentation and macular surgery concepts. It is, therefore, to be expected that more cases will be treated surgically in the near future.

\section{Observation and Natural History}

The cases reported on in the literature illustrate the diversity of outcomes in individual cases. Complete regression of a retinal fold with full restoration of visual function $[6,8-10]$, partial flattening with minimal or moderate improvement of visual function as well as severe permanent structural damage are all possible outcomes if left untreated [8-10]. Unfortunately, there currently seem to be no clinical markers available that would help to predict the outcome, other than anatomical or functional changes over time.

In the majority of reported cases, macular folds will persist and result in permanent visual loss with a visual acuity between counting fingers and 6/24 and associated functional deficiencies, in particular disturbing metamorphopsia [4, 8-10]. In a series of 32 patients reported 
on by Larrison et al. [8], the median final visual acuity was $6 / 24$. In addition, late retinal redetachment, caused by proliferative vitreoretinopathy that might have been triggered by the retinal folds, has been reported in patients with previously stable retinal folds [10].

\section{Surgery for Retinal Folds}

Very few attempts to surgically correct retinal folds have been reported on, including the treatment of folds of other aetiology $[1-3,16,17]$. This contrasts with our impression (based on personal communication at several conferences) that retinal folds still are a clinically relevant complication of retinal detachment surgery, and that more and more of these folds are treated surgically.

There are no clear recommendations on indications for surgery and its timing. In animal experiments, apoptosis of photoreceptors and a thinning of the outer nuclear layer could be seen as early as 1 week after limited macular translocation [12]. An earlier intervention would have a higher chance of restoration of photoreceptor-toretinal pigment epithelium reapposition as well as preservation of photoreceptors and visual function. It may also be easier to unfurl the fold at an earlier stage. However, one of the reasons that often lead to delays to a second intervention is that one may hesitate to perform additional surgery too soon after the first intervention. Moreover, the problem is often diagnosed no earlier than after gas reabsorption. An ambivalent attitude towards an active procedure is also caused by the lack of general recommendations and a possibly challenging surgery on the background that these eyes sometimes show spontaneous resolution and functional improvement $[6,10]$. The few cases of surgical treatment that have been published were operated on up to 3 months following the initial surgery $[2,3]$.

Kertes and Peyman [16] described 2 cases of macular folds following vitrectomy for toxocariasis and proliferative diabetic retinopathy. The cases were treated by peeling of all epiretinal membranes, and the folds were then prodded and massaged under heavy liquid using a silicone-tipped cannula. Herbert et al. [3] employed a modified macula relocation technique. They initially detached the retina in the area of the fold, using a subretinal infusion with a 41-gauge cannula. The authors then installed a short-term perfluorohexyloctane $\left(\mathrm{F}_{6} \mathrm{H}_{8}\right)$ tamponade. This resulted in successful unfolding of the retina without the need for direct surgical manipulation of the fold. A similar technique using $\mathrm{SF}_{6}$ instead of heavy liquid tamponade was successfully employed by El-Amir et al. [2]. One of the authors of this article (S.B.) has used this technique to treat 3 cases of macular folds successfully [unpubl. data]. Trinh et al. [1] also performed a limited retinal redetachment with subretinal infusion of balanced salt solution and fluid air exchange. They then used silicone oil as a tamponade agent and, in addition, applied laser photocoagulation to the edge of the fold after its unfolding in order to fixate the unfolded retina [1]. Kadonosono et al. [17] described the surgical correction of a macular fold following limited macular translocation. This could be achieved by release of the scleral shortening and repeated gas injection to unfold the retina [17].

The cases are to few in number to draw any conclusions other than that an unfolding of retinal tissue can be achieved by a secondary surgical intervention. Herbert et al. [3] reported a functional improvement of 2 lines of Snellen visual acuity, accompanied by a significant reduction of visual disturbances. El-Amir et al. [2] could achieve a significant improvement in visual acuity from $1 / 60$ to $6 / 12$ postoperatively. Similarly, Trinh et al. [1] described an improvement in visual acuity from 20/320 to 0.2 following their intervention [1]. Again, the extremely varying natural course has to be considered when interpreting these results. Severe complications (e.g. retinal tearing or macular holes during forced induction of retinal redetachment) did not occur in these few case reports that have been published.

\section{Conclusion}

Macular folds are a rare but clinically important complication of retinal detachment surgery. It is crucial to identify patients at risk. In the majority of such cases, precautionary intraoperative manipulations and appropriate postoperative posture will avoid the development of macular folds. Once they occurred, we recommend surgical treatment to unfold the retina in cases with significant visual disturbances. We currently perform a technique similar to the ones described in the literature with induction of a localised redetachment through subretinal infusion of balanced solution with a 41-gauge cannula, followed by fluid air exchange, gas tamponade and facedown posture during the immediate postoperative period [2].

\section{Disclosure Statement}

The authors attest that they have no conflict of interest associated with the contents of this paper.

Ophthalmologica 2011;226(suppl 1):18-26 


\section{References}

$>1$ Trinh L, Glacet-Bernard A, Colasse-Marthelot V, Leynaud JL, Soubrane G: Macular fold following retinal detachment surgery (in French). J Fr Ophtalmol 2006;29:995-999.

$>2$ El-Amir AN, Every S, Patel CK: Repair of macular fold following retinal reattachment surgery. Clin Experiment Ophthalmol 2007; 35:791-792.

3 Herbert EN, Groenewald C, Wong D: Treatment of retinal folds using a modified macula relocation technique with perfluoro-hexyloctane tamponade. Br J Ophthalmol 2003; 87:921-922.

$\checkmark 4$ Pierro L, Sadda SR, Gagliardi M, Mantovani E, de Benedetto U, Codenotti M, Bandello F: SD OCT features of dry arcuate longstanding retinal folds. Eur J Ophthalmol 2011;21: 215-217.

$>5$ Pavan PR: Retinal fold in macula following intraocular gas: an avoidable complication of retinal detachment surgery. Arch Ophthalmol 1984;102:83-84.

6 Ruiz-Moreno JM, Montero JA: Sliding macular fold following retinal detachment surgery. Graefes Arch Clin Exp Ophthalmol 2011;249:301-303.
7 Twomey JM, Leaver PK: Retinal compression folds. Eye (Lond) 1988;2(pt 3):283-287.

$\checkmark 8$ Larrison WI, Frederick AR Jr, Peterson TJ, Topping TM: Posterior retinal folds following vitreoretinal surgery. Arch Ophthalmol 1993;111:621-625.

$\checkmark 9$ Lewen RM, Lyon CE, Diamond JG: Scleral buckling with intraocular air injection complicated by arcuate retinal folds. Arch Ophthalmol 1987;105:1212-1214.

10 van Meurs JC, Humalda D, Mertens DA, Peperkamp E: Retinal folds through the macula. Doc Ophthalmol 1991;78:335-340.

11 Shiragami C, Shiraga F, Yamaji H, Fukuda K, Takagishi M, Morita M, Kishikami T: Unintentional displacement of the retina after standard vitrectomy for rhegmatogenous retinal detachment. Ophthalmology 2010; 117:86-92e1.

12 Hayashi A, Usui S, Kawaguchi K, Fujioka S, Kusaka S, Fujikado T, Ohji M, Tano Y: Retinal changes after retinal translocation surgery with scleral imbrication in dog eyes. Invest Ophthalmol Vis Sci 2000;41:4288-4292.
13 Wong D, Williams RL, German MJ: Exchange of perfluorodecalin for gas or oil: a model for avoiding slippage. Graefes Arch Clin Exp Ophthalmol 1998;236:234-237.

14 Fujii GY, Pieramici DJ, Humayun MS, Schachat AP, Reynolds SM, Melia M, de Juan E Jr: Complications associated with limited macular translocation. Am J Ophthalmol 2000; 130:751-762.

15 Brazitikos PD, Androudi S, D’Amico DJ, Papadopoulos N, Dimitrakos SA, Dereklis DL, Alexandridis A, Lake S, Stangos NT: Perfluorocarbon liquid utilization in primary vitrectomy repair of retinal detachment with multiple breaks. Retina 2003;23. 615-621.

16 Kertes PJ, Peyman GA: Management of dry retinal folds. Int Ophthalmol 1997;21:5355.

17 Kadonosono K, Takeuchi S, Iwata S, Uchio E, Itoh N, Akura J: Macular fold after limited macular translocation treated with scleral shortening release and intravitreal gas. Am J Ophthalmol 2001;132:790-792. 